\title{
Numerical study on the interaction between non-linear wave, buried pipeline and non-homogenous porous seabed
}

\author{
F.P. Gao ${ }^{\mathrm{a}, *}$, D.S. Jeng ${ }^{\mathrm{b}}$, H. Sekiguchi ${ }^{\mathrm{c}}$ \\ a'Institute of Mechanics, Chinese Academy of Science, Beijing 100080, People's Republic of China \\ ${ }^{\mathrm{b}}$ School of Engineering, Griffith University Gold Coast Campus, QLD 9726, Australia \\ ${ }^{\mathrm{c}}$ Disaster Orevention Research Institute, Kyoto University, Uji, Kyoto 611, Japan
}

Received 12 February 2002; received in revised form 31 January 2003; accepted 25 February 2003

\begin{abstract}
The wave-seabed-pipeline interaction problem is particularly important for coastal geotechnical engineers involved in the design of submarine pipelines. Most previous investigations have only concerned with the soil response due to linear progressive ocean waves, even though strong evidence of wave non-linearity has been reported in the literature. In this study, the finite element model (GFEM-WSSI) proposed by the first author is adopted to investigate the interaction between nonlinear ocean waves, a buried pipelines and a porous seabed. Unlike previous models, the deformation of pipeline and non-homogeneous soil behavior are also considered in the new model. Numerical results demonstrate that the influence of non-linear wave components can not always be ignored without committing substantial error, especially for the case of large waves in shallow water. Also, revealed is that nonlinear wave-induced seabed response is affected significantly by variable permeability and shear modulus. Moreover, the internal stresses of buried pipeline increase significantly with a decrease of pipeline thickness.
\end{abstract}

(C) 2003 Elsevier Science Ltd. All rights reserved.

Keywords: Non-linear waves; Wave-seabed-pipeline interaction; Finite element model

\section{Introduction}

Submarine pipelines have been a type of commonused offshore installations. It has been widely used to transport natural oil, gas, and industrial waste water etc. When gravitational waves propagate over the ocean, they cause fluctuating pressure upon the seabed, which will further induce excess pore pressure and effective stresses within seabed soil. The excess pore pressure may be large enough to induce shear failure or liquefaction of the soil around pipelines. Under such conditions, the pipelines will loss stability, which will cause economic losses and environmental pollution. Therefore, the evaluation of the wave-induced soil response around a buried pipeline (including pore pressure and effective stresses etc.) is important for coastal geotechnical engineers.

* Corresponding author. Tel.: +61-7-5552-8683; fax: +61-7-55528065 .

E-mail address: d.jeng@griffith.edu.au (D.S. Jeng).
To date, many researchers have developed theories for the wave-induced soil responses in an elastic medium. Yamamoto et al. [27] and Madsen [15] proposed analytical solutions for ocean wave-seabed interaction problem within hydraulically isotropic and anisotropic uniform seabed of infinite thickness, respectively. Mei and Foda [20] proposed a boundary-layer approximation for the wave-induced soil response. Besides the development of analytical solutions, numerical simulations have been widely applied to examine such a problem in recent years, such as the finite difference method $[16,28]$, the finite element method $[5,6,12,24,25]$ and the boundary element method [21]. However, all aforementioned investigations have only examined the soil response in a pro-elastic seabed under the action of twodimensional progressive waves without the presence of marine structures.

Although the importance of wave-seabed-pipeline interaction phenomenon has been addressed in the literature [4], this problem has not been fully understood because of the complication of the behaviors of soil, wave loading and geometry of the pipeline. Based on 
the potential theory, the hydrodynamic uplift forces on the buried pipelines has been studied $[13,14,23]$. However, the potential theory is far from the realistic conditions of the soil and pore-fluid two-phase medium. Furthermore, all these theories provide no information for the effective stresses and soil displacements in the seabed.

Based on Biot's theory [1], the wave-induced pore pressure around a buried pipeline has been studied through a boundary integral equation method [3] and a finite element method [17-19]. Among these, Cheng and Liu [3] considered a buried pipe in a region that is surrounded by two impermeable walls. Magda [17-19] considered a similar case with a wider range of the degree of saturation. All these have only discussed the wave-induced pore pressure and uplift forces around the buried pipe. Other soil responses in the vicinity of a buried pipeline, such as effective stresses, were not discussed. Jeng [10] investigated the wave-induced pore pressure, effective stresses along the buried pipeline in the seabed with variable permeability and shear modulus. But, the author has only concerned with the soil responses due to linear wave, ignoring the influence of wave non-linearity. In the previous investigations for the wave-seabed interaction [26], the effect of wave nonlinearity has been found not being neglected without substantial error, especially for large waves in shallow water. Therefore, it is necessary to examine the influence of non-linear wave components upon the wave-pipeline-seabed interaction.

This paper is aimed at investigating the distribution of non-linear wave-induced pore pressure along the surface of pipeline and the internal stresses within the pipeline in non-homogenous seabed. In this study, the finite element model (GFEM-WSSI) proposed by the first author [11] will be adopted. The numerical results of soil and pipeline responses under the non-linear wave loading will be compared with that under linear wave loading.

\section{Boundary value problem}

In this study, we consider a fully buried pipeline (with radius of $R$ ) in a porous seabed of finite thickness $h$ laid upon an impermeable rigid bottom, as depicted in Fig. 1. The wave is assumed to propagate in the positive $x$-direction, while the $z$-direction is upward from the interface between porous seabed and impermeable rigid bottom (see Fig. 1).

\subsection{Governing equations}

\subsubsection{Governing equations for porous flows in a seabed}

In this study, the consolidation Eq. (1), which was extended from Terzaghi's theory and has been generally accepted as the governing equation for flow of a compressible pore fluid in a compressible pore medium, is

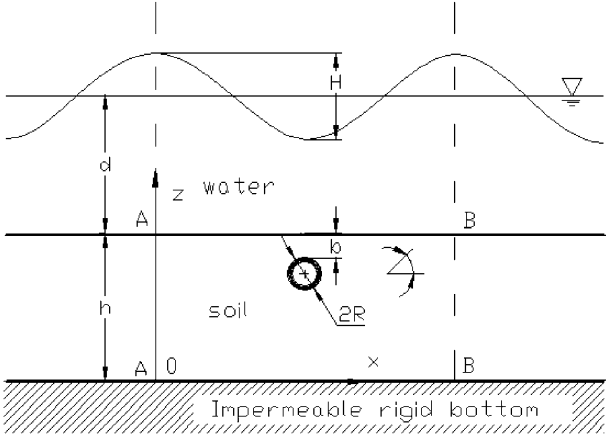

Fig. 1. Definition of wave-seabed-pipe interaction system.

adopted to treat the wave-seabed interaction with variable permeability as

$K(z) \nabla^{2} p+\frac{\mathrm{d} K(z)}{\mathrm{d} z} \frac{\partial p}{\partial z}-\gamma_{\mathrm{w}} n \beta \frac{\partial p}{\partial t}=\gamma_{\mathrm{w}} \frac{\partial}{\partial t}\left(\nabla \cdot \vec{u}_{\mathrm{s}}\right)$,

where $K$ is soil permeability, $p$ is pore pressure, $\gamma_{\mathrm{w}}$ is the unit weight of pore water, $n$ is soil porosity, $t$ is the time, $\vec{u}_{\mathrm{s}}$ is the vector of soil displacements, $\beta$ is the compressibility of pore fluid, defined as

$\beta=\frac{1}{K_{\mathrm{wo}}}+\frac{1-S}{P_{\text {wo }}}$,

in which, $K_{w}$ is the true modulus of elasticity of water (taken as $2 \times 10^{9} \mathrm{~N} / \mathrm{m}^{2}$ ), $P_{\text {wo }}$ is the absolute water pressure and $S$ is the degree of saturation.

Under conditions of plane strain, the stress-strain relationship can be expressed as

$\sigma_{x}^{\prime}=2 G(z)\left[\frac{\partial u_{\mathrm{s}}}{\partial x}+\frac{\mu}{1-2 \mu}\left(\nabla \cdot \vec{u}_{\mathrm{s}}\right)\right]$,
$\sigma_{z}^{\prime}=2 G(z)\left[\frac{\partial w_{\mathrm{s}}}{\partial z}+\frac{\mu}{1-2 \mu}\left(\nabla \cdot \vec{u}_{\mathrm{s}}\right)\right]$,

$\tau_{x z}=G(z)\left[\frac{\partial u_{\mathrm{s}}}{\partial z}+\frac{\partial w_{\mathrm{s}}}{\partial x}\right]=\tau_{z x}$,

where $G$ is the shear modulus of soil, which is related to Young's modulus $(E)$ and Poisson's ratio $(\mu)$ as $E / 2(1+\mu)$. It is noted that a positive sign is taken for a compressive normal stress in this study.

Neglecting the effects of body forces and inertia terms, the equations governing the overall equilibrium of a porous medium can be expressed in terms of pore pressure and soil displacements as,

$$
\begin{aligned}
& G(z) \nabla^{2} u_{\mathrm{s}}+\frac{G}{1-2 \mu} \frac{\partial}{\partial x}\left(\nabla \cdot \vec{u}_{\mathrm{s}}\right)+\frac{\mathrm{d} G}{\mathrm{~d} z}\left(\frac{\partial u_{\mathrm{s}}}{\partial z}+\frac{\partial w_{\mathrm{s}}}{\partial x}\right) \\
& =-\frac{\partial p}{\partial x},
\end{aligned}
$$




$$
\begin{aligned}
& G(z) \nabla^{2} w_{\mathrm{s}}+\frac{G}{1-2 \mu} \frac{\partial}{\partial z}\left(\nabla \cdot \vec{u}_{\mathrm{s}}\right)+\frac{\mathrm{d} G}{\mathrm{~d} z} \\
& \times\left(\mu \frac{\partial u_{\mathrm{s}}}{\partial z}+(1-\mu) \frac{\partial w_{\mathrm{s}}}{\partial x}\right) \\
& =-\frac{\partial p}{\partial z}
\end{aligned}
$$

in the $x$ and $z$ directions, respectively.

\subsubsection{Governing equations for buried pipe}

In this study, we consider the buried pipe as an elastic material. With the absence of the body forces, based on the minimum potential energy theorem, the total potential energy of the buried pipe can be expressed as

$\Pi=\frac{1}{2} \int_{V} \sigma_{\mathrm{p}, i j} \varepsilon_{\mathrm{p}, i j} \mathrm{~d} V-\int_{A} f_{i} u_{\mathrm{p}, i} \mathrm{~d} A$.

in which $\sigma_{\mathrm{p}}$ and $\varepsilon_{\mathrm{p}}$ are internal stress and strain within pipeline wall respectively, $f$ is the wave-induced forces acting on the buried pipeline, and $u_{\mathrm{p}}$ is the pipeline deformation induced by external forces. Applying the variational method to $(5)$, we have

$\delta \Pi=\frac{1}{2} \int_{V} \sigma_{\mathrm{p}, i j} \delta \varepsilon_{\mathrm{p}, i j} \mathrm{~d} V-\int_{A} f_{i} \delta u_{\mathrm{p}, i} \mathrm{~d} A$.

According to the minimum energy theorem (i.e., $\delta \Pi=0)$, can be written as

$\frac{1}{2} \int_{V} \sigma_{\mathrm{p}, i j} \delta \varepsilon_{\mathrm{p}, i j} \mathrm{~d} V=\int_{A} f_{i} \delta u_{\mathrm{p}, i} \mathrm{~d} A$.

As the first approximation, we assume no relative movement between pipe and soil particles. That is, the soil displacements are assumed to be in conformity with the displacement of pipeline, i.e., the condition of displacement continuity is applied. This assumption is reasonable for the pipelines whose steel wall is covered with concrete coating. The rough concrete material will be conformable with the relative stiff soils, such as medium and desne silt, whose deformation is small. Moreover, the concrete coating will creates an impermeable boundary conditions, thus no flux takes place along the pipeline surface, which will be discussed in Section 2.2.

Under the above assumptions, the wave-induced soil deformation is also included in terms of pore pressure by (1), (4a) and (4b). Also, the shear stress and normal stresses are linked with soil displacements by (3a), (3b) and (3c). Thus, while we solve the soil response around the pipeline with internal stresses, the shear stress, normal stresses and soil displacements are transferred (they are directly affected by pore pressure) into pore pressures as the external loading in the evaluation of the internal stresses within the pipeline.

\subsection{Boundary conditions}

For a porous seabed of finite thickness, as shown in Fig. 1, the evaluation of the wave-induced seabed response requires the solution of (1), (4a), (4b) and (7), together with the appropriate boundary conditions.

Firstly, for the soil resting on an impermeable rigid base, zero displacements and no vertical flow occurs at the interface between the soil and the impermeable rigid bottom, i.e.

$u_{\mathrm{s}}=w_{\mathrm{s}}=\frac{\partial p}{\partial z}=0, \quad$ at $z=0$.

Secondly, we assume that the bottom frictional stress is small and negligible. The vertical effective normal stress and shear stress vanish at the surface of seabed,

$\sigma_{z}^{\prime}=\tau_{x z}=0, \quad$ at $z=h$.

Thirdly, the pore pressure at the surface of the seabed is equal to the wave pressure induced by the progressive wave. It has been agreed that the wave profile has steep crest and flat trough in shallow water, result from its strong non-linearity. Therefore, the validity of the conventional Stokes' wave theories in shallow water has been doubted. As Huang et al. [9] and Sobey et al. [22] pointed out, Stokes' wave theories may not be very suitable for a wave in shallow water near the breaking line. However, it is acceptable under most wave conditions for engineering applications. In fact, engineering practice in recent years has still applied heavily on the Stokes' wave theories for it performs the basic characteristics of wave non-linearity and its accuracy satisfies requirements of most engineering problems. In this study, based on the non-linear wave theory [8], thirdorder approximation of non-linear wave pressure in two-dimensional problems are employed,

$p=p_{\mathrm{a}} \cos (k x-\omega t)+p_{2 \mathrm{nd}}+p_{3 \mathrm{rd}}, \quad$ at $z=h$.

where $p_{\mathrm{a}}$ is the wave pressure of the first-order (linear) of the short-crested wave theory, $p_{2 \text { nd }}$ and $p_{3 \text { rd }}$ are the second and third-order components, respectively, which are given by

$p_{\mathrm{a}}=\frac{\gamma_{\mathrm{w}} H}{2 \cosh (k d)}$,

$p_{2 \mathrm{nd}}=\left(\frac{k H}{2}\right)^{2} \frac{\gamma_{\mathrm{w}}}{k}\left[A_{21}+A_{22} \cos 2(k w-\omega t)\right]$,

$$
\begin{aligned}
p_{3 \mathrm{rd}}= & \frac{1}{2}\left(\frac{k H}{2}\right)^{3} \frac{\gamma_{\mathrm{w}}}{k} \\
& \times\left[A_{31} \cos (k x-\omega t)+A_{32} \cos 3(k w-\omega t)\right],
\end{aligned}
$$

where, $H$ is wave height, $d$ is water depth, $k$ is wave number ( $=2 \pi / L, L$ is the wave length), $\omega$ is wave frequency 
$(=2 \pi / T, T$ is the wave period),

$A_{21}=-\omega_{\mathrm{o}} \beta_{1}-\frac{\omega_{\mathrm{o}}^{2}}{4 \sinh ^{2} k d}$,

$A_{22}=2 \omega_{\mathrm{o}}\left(\beta_{2}+\beta_{3}\right)-\frac{\omega_{\mathrm{o}}^{2}}{4 \sinh ^{2} k d}$,

$A_{31}=\frac{\omega_{\mathrm{o}}}{\sinh k d}\left(\omega_{1}+2 \beta_{2}+2 \beta_{3}\right)+\beta_{13} \omega_{\mathrm{o}}$,

$A_{32}=\frac{\omega_{\mathrm{o}}}{\sinh k d}\left(\beta_{2}+\frac{1}{2} \beta_{3}\right)+3 \omega_{\mathrm{o}}\left(\beta_{13}+\beta_{33}\right)$,

$\omega_{o}=\sqrt{\tanh k d}$,

$\omega_{1}=\frac{1}{32}\left(2 \omega_{\mathrm{o}}^{5}+59 \omega_{\mathrm{o}}-24 \omega_{\mathrm{o}}^{-3}+27 \omega_{\mathrm{o}}^{-7}\right)$,

$\beta_{1}=\frac{1}{8}\left(-\omega_{\mathrm{o}}^{-3}+\omega_{\mathrm{o}}\right)$

$\beta_{2}=\frac{3\left(\omega_{\mathrm{o}}^{-7}+\omega_{\mathrm{o}}\right)}{16} \cosh (2 k d)=\beta_{3}$,

$\beta_{13}=\frac{\left(2 \omega_{\mathrm{o}}^{5}-13 \omega_{\mathrm{o}}+12 \omega_{\mathrm{o}}^{-3}-9 \omega_{\mathrm{o}}^{-7}\right)}{16 \cosh k d\left(\tanh k d-\tanh ^{-1} k d\right)}$,

$\beta_{31}=\frac{3\left(-5 \omega_{\mathrm{o}}+14 \omega_{\mathrm{o}}^{-3}-9 \omega_{\mathrm{o}}^{-7}\right)}{16 \cosh k d\left(\tanh 3 k d-3 \omega_{\mathrm{o}}^{2}\right)}$,

$\beta_{33}=\frac{\omega_{\mathrm{o}}^{-5}\left(1+3 \omega_{\mathrm{o}}^{4}\right)\left(9 \omega_{\mathrm{o}}^{-8}-22 \omega_{\mathrm{o}}^{-4}+13\right)}{128 \cosh 3 k d}$.

From (11a)-(11c), (10) can be written as

$$
\begin{aligned}
p= & p_{\mathrm{o}}+p_{1} \operatorname{Re}\left\{(\cos k x+i \sin k x) e^{-i \omega t}\right\} \\
& +p_{2} \operatorname{Re}\left\{(\cos 2 k x+i \sin 2 k x) e^{-2 i \omega t}\right\} \\
& +p_{3} \operatorname{Re}\left\{(\cos 3 k x+i \sin 3 k x) \mathrm{e}^{-3 i \omega t}\right\},
\end{aligned}
$$

where

$p_{\mathrm{o}}=\left(\frac{k H}{2}\right)^{2} \frac{\gamma_{\mathrm{w}}}{k} A_{21}$,

$p_{1}=p_{\mathrm{o}}+\frac{1}{2}\left(\frac{k H}{2}\right)^{3} \frac{\gamma_{\mathrm{w}}}{k} A_{31}$, $p_{2}=\left(\frac{k H}{2}\right)^{2} \frac{\gamma_{\mathrm{w}}}{k} A_{22}$,

$p_{3}=\frac{1}{2}\left(\frac{k H}{2}\right)^{3} \frac{\gamma_{\mathrm{w}}}{k} A_{32}$.

Fourthly, it is reasonable to assume there is no flow through the pipeline wall. Thus, the pressure gradient should vanish at the surface of the pipe, i.e.

$\frac{\partial p}{\partial n}=0, \quad$ at $r=\sqrt{\left(x-x_{\mathrm{o}}\right)^{2}+\left(z-z_{\mathrm{o}}\right)^{2}}=R$.

Finally, since the existence of the pipeline only affects the wave-induced soil response near the pipeline, the 'disturbed pressure' from the pipeline should vanish at points far away from the pipe. However, the porous seabed at points is still under wave loading. Thus, the lateral boundary conditions at these points are given by the solution without pipeline, which will be described later.

\section{Finite element formulations}

\subsection{Finite element formulations for the porous flow}

The third-order non-linear wave-induced oscillatory soil response is periodically fluctuating in the temporal domain. Based on (13), the wave-induced pore pressure, soil displacements and effective stresses can be expressed as

$$
\begin{aligned}
\{\Gamma(x, z, ; t)\}= & \left\{\Gamma_{0 \mathrm{r}}(x, z, ; t)\right\} \\
& +\left[\left\{\Gamma_{1 \mathrm{r}}(x, z, ; t)\right\}+i\left\{\Gamma_{1 \mathrm{c}}(x, z, ; t)\right\}\right] \mathrm{e}^{-i \omega t} \\
& +\left[\left\{\Gamma_{2 \mathrm{r}}(x, z, ; t)\right\}+i\left\{\Gamma_{2 \mathrm{c}}(x, z, ; t)\right\}\right] \mathrm{e}^{-2 i \omega t} \\
& +\left[\left\{\Gamma_{3 \mathrm{r}}(x, z, ; t)\right\}+i\left\{\Gamma_{3 \mathrm{c}}(x, z, ; t)\right\}\right] \mathrm{e}^{-3 i \omega t},
\end{aligned}
$$

$\Gamma=\left[\begin{array}{llllll}p & u_{\mathrm{s}} & w_{\mathrm{s}} & \sigma_{\mathrm{s} x}^{\prime} & \sigma_{\mathrm{s} z}^{\prime} & \tau_{\mathrm{s} x z}\end{array}\right]^{T}$,

where subscripts ' $r$ ' and ' $c$ ' represent the real and imaginary parts of the soil response, respectively. Substituting (17) into (1), (4a) and (4b), then directly applying the Galerkin method [30], to these equations, the finite element analytical formulations can be expressed in a matrix form as

$$
\begin{aligned}
\int_{\mathrm{s}} N_{i}\left[Q_{e}\right] \mathrm{d} S & =\int_{V} B_{1}^{T} D_{1} B_{1} \mathrm{~d} V[P] \\
& +\int_{V} B_{2}^{T} D_{2} B_{2} d V[P] \\
& +\int_{V} B_{3}^{T} D_{3} B_{3} d V\left[U_{\mathrm{s}}\right],
\end{aligned}
$$


$\int_{\mathrm{s}} N_{i}\left[F_{e}\right] \mathrm{d} S=\int_{V} B_{4}^{T} D_{4} B_{4} \mathrm{~d} V\left[U_{\mathrm{s}}\right]+\int_{V} B_{5}^{T} B_{1} d V[P]$,

where,

$$
\begin{aligned}
& {\left[Q_{\mathrm{e}}\right]=\left[\begin{array}{lllll}
\left(q_{n \mathrm{r}}\right)_{1} & 0 & \cdots & \left(q_{n \mathrm{r}}\right)_{n_{\mathrm{e}}} & 0 \\
0 & \left(q_{n \mathrm{c}}\right)_{1} & \cdots & 0 & \left(q_{n \mathrm{c}}\right)_{n_{\mathrm{e}}}
\end{array}\right],} \\
& {\left[F_{\mathrm{e}}\right]=} \\
& {\left[\begin{array}{lllllll}
\left(f_{x \mathrm{r}}\right)_{1} 0 & \left(f_{y \mathrm{r}}\right)_{1} 0 & \cdots & \left(f_{x \mathrm{r}}\right)_{n_{\mathrm{e}}} 0 & \left(f_{y \mathrm{r}}\right)_{n_{\mathrm{e}}} & 0 \\
0 & \left(f_{x \mathrm{c}}\right)_{1} 0 & \left(f_{y \mathrm{c}}\right)_{1} \cdots & 0 & \left(f_{x \mathrm{c}}\right)_{n_{\mathrm{e}}} 0 & \left(f_{y \mathrm{c}}\right)_{n_{\mathrm{e}}}
\end{array}\right],} \\
& {\left[\left\{\begin{array}{c}
q_{n \mathrm{r}} \\
q_{n \mathrm{c}}
\end{array}\right\}\right]=\left[\frac{K}{\gamma_{\mathrm{w}}}\left\{\begin{array}{l}
\frac{\partial p_{\mathrm{r}}}{\partial x} \\
\frac{\partial p_{\mathrm{c}}}{\partial x}
\end{array}\right\} n_{x}+\frac{K}{\gamma_{\mathrm{w}}}\left\{\begin{array}{l}
\frac{\partial p_{\mathrm{r}}}{\partial z} \\
\frac{\partial p_{\mathrm{c}}}{\partial z}
\end{array}\right\} n_{z}\right],} \\
& {\left[\left\{\begin{array}{l}
f_{x \mathrm{r}} \\
f_{x \mathrm{c}} \\
f_{z \mathrm{r}} \\
f_{z \mathrm{c}}
\end{array}\right\}\right]=\left[\left\{\begin{array}{c}
S_{\mathrm{s} x \mathrm{r}} \\
S_{\mathrm{s} x \mathrm{c}} \\
T_{\mathrm{sr}} \\
T_{\mathrm{sc}}
\end{array}\right\} n_{x}+\left\{\begin{array}{l}
T_{\mathrm{sr}} \\
T_{\mathrm{sc}} \\
S_{\mathrm{s} x \mathrm{r}} \\
S_{\mathrm{s} x \mathrm{c}}
\end{array}\right\} n_{z}\right] \text {, }} \\
& {[P]=\left[\begin{array}{lllll}
\left(p_{\mathrm{r}}\right)_{1} & 0 & \cdots & \left(p_{\mathrm{r}}\right)_{n_{\mathrm{e}}} & 0 \\
0 & \left(p_{\mathrm{c}}\right)_{1} & \cdots & 0 & \left(p_{\mathrm{c}}\right)_{n_{\mathrm{e}}}
\end{array}\right],} \\
& {\left[U_{\mathrm{s}}\right]=} \\
& {\left[\begin{array}{lllllll}
\left(u_{\mathrm{sr}}\right)_{1} 0 & \left(w_{\mathrm{sr}}\right)_{1} 0 & \cdots & \left(u_{\mathrm{sr}}\right)_{n_{\mathrm{e}}} 0 & \left(w_{\mathrm{sr}}\right)_{n_{\mathrm{e}}} 0 \\
0 & \left(u_{\mathrm{sc}}\right)_{1} 0 & \left(w_{\mathrm{sc}}\right)_{1} \cdots & 0 & \left(u_{\mathrm{sc}}\right)_{n_{\mathrm{e}}} & \left(w_{\mathrm{sc}}\right)_{n_{\mathrm{e}}}
\end{array}\right] \text {, }}
\end{aligned}
$$

where $n_{\mathrm{e}}$ is the number of nodes per element, $N_{i}$ is the shape function of the $i$-th node, $p_{\mathrm{r}}, u_{\mathrm{s}}$ and $w_{\mathrm{s}}$ are in form of (16), and coefficient matrices $B_{i}$ and $D_{i}$ are given in the Appendix.

\subsection{Finite element formulations for the buried pipe}

Similarly, the third-order non-linear wave-induced internal stresses and deformations of the pipe and the external forces can be expressed as

$$
\begin{aligned}
\{\Omega(x, z, ; t)\}= & \left\{\Omega_{0 r}(x, z, ; t)\right\} \\
& +\left[\left\{\Omega_{1 \mathrm{r}}(x, z, ; t)\right\}+i\left\{\Omega_{1 \mathrm{c}}(x, z, ; t)\right\}\right] \mathrm{e}^{-i \omega t} \\
& +\left[\left\{\Omega_{2 \mathrm{r}}(x, z, ; t)\right\}+i\left\{\Omega_{2 \mathrm{c}}(x, z, ; t)\right\}\right] \mathrm{e}^{-2 i \omega t} \\
& +\left[\left\{\Omega_{3 \mathrm{r}}(x, z, ; t)\right\}+i\left\{\Omega_{3 \mathrm{c}}(x, z, ; t)\right\}\right] \mathrm{e}^{-3 i \omega t},
\end{aligned}
$$

$$
\Omega=\left[\begin{array}{llllll}
f_{\mathrm{p}} & u_{\mathrm{p}} & w_{\mathrm{p}} & \sigma_{\mathrm{p} x}^{\prime} & \sigma_{\mathrm{p} z}^{\prime} & \tau_{\mathrm{p} x z}
\end{array}\right]^{T} .
$$

$$
\int_{S} N_{i}\left[F_{\mathrm{p}}\right] t_{\mathrm{p}} \mathrm{d} S=\int_{A} B_{5}^{T} D_{5} B_{5} t_{\mathrm{p}} \mathrm{d} A\left[U_{\mathrm{p}}\right],
$$

where $U_{\mathrm{p}}$ is the nodal displacement matrix of pipe, $t_{\mathrm{p}}$ is the thickness of the pipeline and $F_{\mathrm{p}}$ is the force matrix acting on the pipe, and the matrices $D_{1}-D_{5}$ are given in the Appendix.

\subsection{Numerical procedure}

The first step in solving the wave-soil-pipe interaction with both variable permeability and shear modulus is to establish the lateral boundary conditions. To do so, the wave-seabed interaction can be solved by employing the principle of repeatability [29]. As shown in Fig. 1, the wave-induced pore pressure and soil displacements at sections AA and BB should be identical, because the seabed is under a periodical loading. That is,

$$
\begin{aligned}
& p(x=A A, z ; t)=p(x=B B, z ; t) \\
& u_{\mathrm{s}}(x=A A, z ; t)=u_{\mathrm{s}}(x=B B, z ; t) \\
& w_{\mathrm{s}}(x=A A, z ; t)=w_{\mathrm{s}}(x=B B, z ; t)
\end{aligned}
$$

This concept is particularly convenient for periodical loading such as the present problem.

Once the latter boundary conditions are obtained, the whole wave-seabed-pipe problem can be solved. Because a concentration of stresses is to be expected, the local refinement of the FE (finite element) mesh always has to be taken into account in the region near a structure. To improve the accuracy of the solution in this region, we use two different mesh systems in the present model. As seen in Fig. 2, an eight-node iso-parametric element is used in the region near the pipeline. Outside this region, an eight-nodal rectangular element is used. This kind of mesh has been used for treating the problem around a pipe-like structure [18].

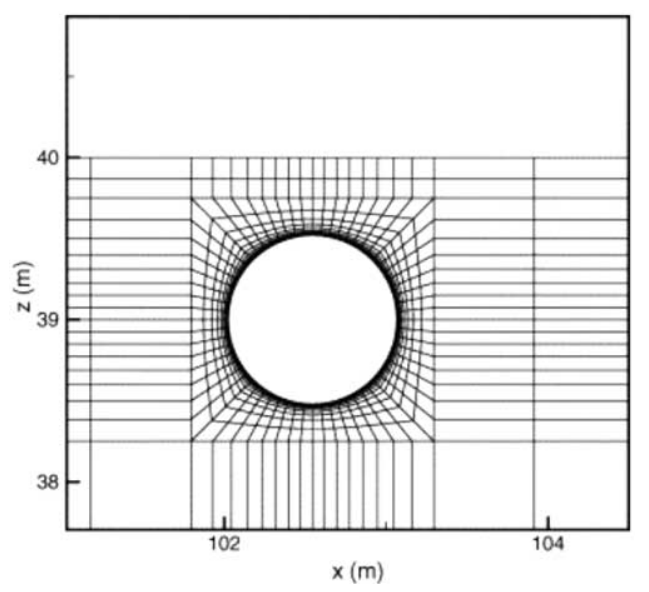

Fig. 2. Finite element mesh in the vicinity of the pipeline. 
Table 1

Input data in numerical examples

\section{Wave characteristics}

Wave period $(T)$

Water depth $(d)$

Wavelength $(L)$

Wave height $(H)$

Soil characteristics

Seabed thickness $(h)$

Poisson ratio (v)

Porosity (n)

Shear modulus $\left(G_{\mathrm{o}}\right)$

Permeability $\left(K_{\mathrm{o}}\right)$

Degree of saturation $(S)$

Pipe characteristics

Burial depth (b)

Poisson ratio $(v)$

Young's modulus $\left(E_{\mathrm{p}}\right)$

Pipe radius $(R)$

Pipe thickness $\left(t_{\mathrm{p}}\right)$

Density of pipe $\left(\rho_{\mathrm{p}}\right)$
$12.5 \mathrm{~s}$

$40(\mathrm{~m})$ (various in Section 4.1)

205 (m)

10 (m) (various in Section 4.1)

$40(\mathrm{~m})$

0.4

0.4

$2.5 \times 10^{7}(\mathrm{~Pa})$

$10^{-2} \mathrm{~m} / \mathrm{s}$

1.0

$1.0(\mathrm{~m})$

0.2

$3.0 \times 10^{10}(\mathrm{~Pa})$

$0.52(\mathrm{~m})$

$0.02(\mathrm{~m})$ (various in Section 4.2.4) $2400\left(\mathrm{~kg} / \mathrm{m}^{3}\right)$

\section{Numerical results and discussion}

\subsection{Effects of non-linear wave components}

The first aim of this paper concerns with how the nonlinear wave components affect the soil responses around the buried pipe. The influences of wave non-linearity on the wave kinematics and dynamics have been well documented in the literature $[9,22]$. The influences of non-linear waves on the response of seabed with absence of structures were investigated in the past [26]. However, the effects of non-linear wave component on wave-soil-pipe interaction have not been examined until now.

Marine sediments below water-soil interface undergo consolidation due to both the overburden soil pressure and the wave pressure above them. For normally consolidated sediments of sandy seabed, soil parameters are depth-dependent. Normally speaking, with increasing
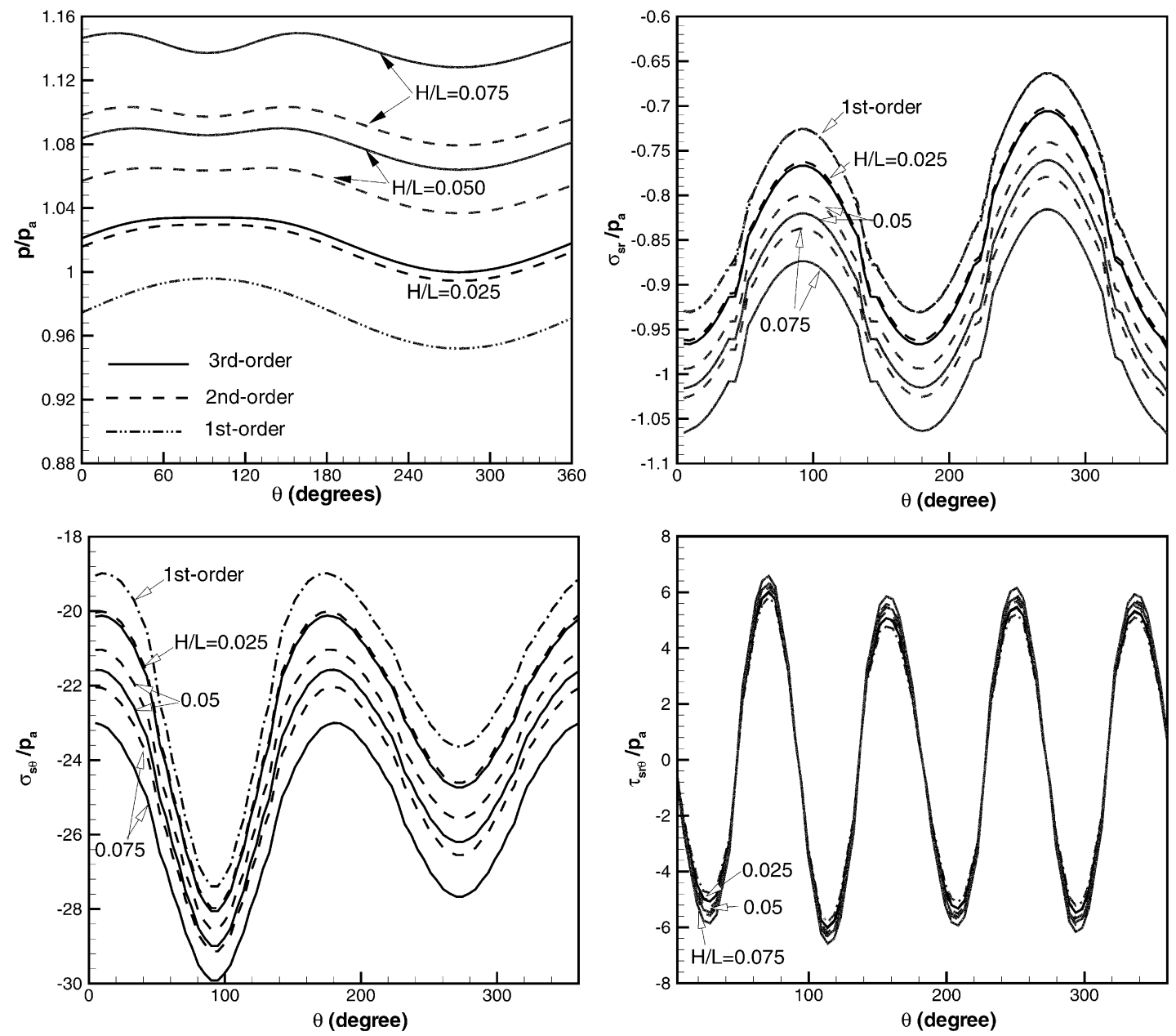

Fig. 3. Distribution of wave-induced pore pressure, normal stresses and shear stress along pipe surface for various wave steepness with different order solutions $(d=40 \mathrm{~m})$. 
depth, the submerged unit weight and the porosity decrease, thereby the permeability increase and the shear modulus decrease. An example of permeability varying with burial depth was reported for the marine sediments in the Gulf of Mexico [2]. For consolidation problem, the soil whose shear modulus increases linearly with depth (so called Gibson Soil) has been studied by Gibson [7], without water wave loading.

To examine the effects of non-linear wave component on buried pipeline responses, a kind of Gibson Soil will be used, i.e. the depth function for shear modulus is chosen as

$G=G_{\mathrm{o}}[1+9(1-z / h)]$,

and the depth function for permeability is supposed to reduce exponentially,

$K=K_{\mathrm{o}} \exp [-2.3025(1-z / h)]$.

Other input date for the parameters of wave, soil and pipe are listed in Table 1.

Fig. 3 illustrates the distribution of non-linear waveinduced pore pressure $\left(p / p_{\mathrm{a}}\right)$, effective normal stresses
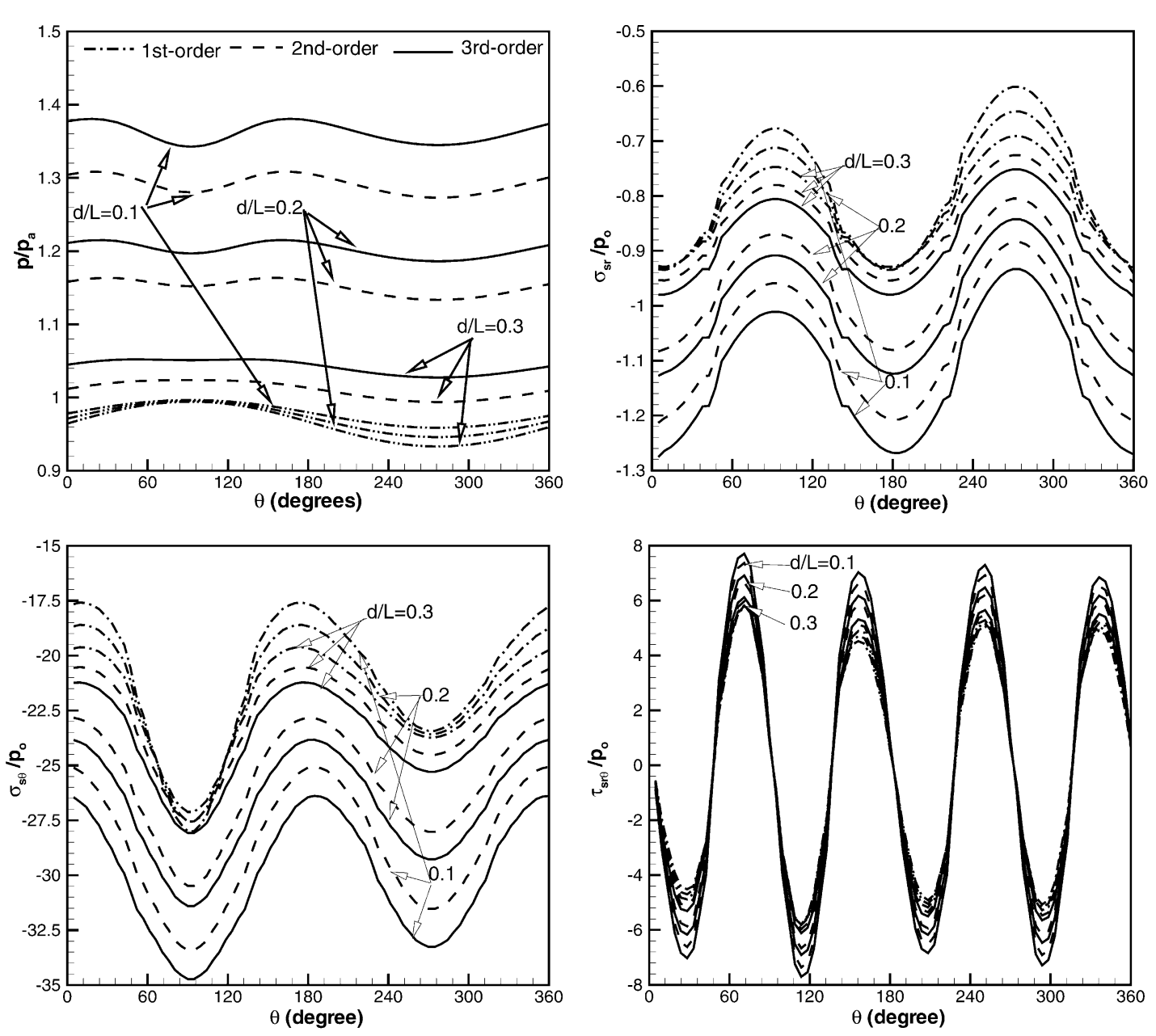

$\left(\sigma_{\mathrm{sr}} / p_{\mathrm{a}}\right.$ and $\left.\sigma_{\mathrm{s} \theta} / p_{\mathrm{a}}\right)$ and shear stress $\left(\tau_{\mathrm{sr} \theta} / p_{\mathrm{a}}\right)$ along pipe surface for various wave steepness $(H / L)$ with different order solutions. The figure shows that, with the increase of wave steepness, the wave induced pore pressure and effective normal stresses increase obviously, but the shear stress increases slightly. The effects of secondorder and third-order components increase as the wave steepness increases. Furthermore, the relative deference of seabed response due to non-linear wave and linear wave also increase as the steepness increase. In addition, the profile of pore pressure at the upper side of pipe (i.e. $0^{\circ}<\theta<180^{\circ}$ ) induced by non-linear wave is much different from that by linear wave. This implies that the effects of non-linear wave components on the response of soil become significant at the upper side of pipe.

Fig. 4 shows the relationship of non-linear waveinduced pore pressure $\left(p / p_{\mathrm{a}}\right)$, effective normal stresses $\left(\sigma_{\mathrm{sr}} / p_{\mathrm{a}}\right.$ and $\left.\sigma_{\mathrm{s} \theta} / p_{\mathrm{a}}\right)$ and shear stress $\left(\tau_{\mathrm{sr} \theta} / p_{\mathrm{a}}\right)$ along pipe surface for various relative water depths $(d / L)$ with different order solutions. The figure clearly shows that the wave induced pore pressure, normal stresses and shear stress increase as relative water depth decreases. Moreover, the

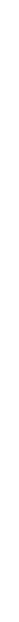

Fig. 4. Distribution of non-linear wave-induced pore pressure, normal stress and shear stress along pipe surface for various wave relative water depth with different order solutions $(H=10 \mathrm{~m})$. 
relative deference of wave induced responses between different order solutions increase as relative water depth decreases. This indicates that the non-linear wave induced soil and pipe responses are higher in shallow water than in deep water.

From the above analysis, it can be concluded that, the non-linear wave effects on soil and buried pipe responses become more obvious, with the decrease relative water depth $(d / L)$ or with the increase of wave steepness $(H / L)$. The non-linear wave effects are not ignorable, especially for high wave steepness in shallow water.

Table 2

Depth function of permeability and shear modulus

\begin{tabular}{lll}
\hline $\begin{array}{l}\text { Depth } \\
\text { function }\end{array}$ & Variable permeability & Variable shear modulus \\
\hline$K_{1}$ or $G_{1}$ & $K=K_{\mathrm{o}}$ & $G=G_{\mathrm{o}}$ \\
$K_{2}$ or $G_{2}$ & $K=K_{\mathrm{o}} \exp [-2.3025(1-z / h)]$ & $G=G_{\mathrm{o}} \exp [-2.3025(1-z / h)]$ \\
$K_{3}$ or $G_{3}$ & $K=K_{\mathrm{o}} \exp [-4.6050(1-z / h)]$ & $G=G_{\mathrm{o}} \exp [-4.6050(1-z / h)]$ \\
$K_{4}$ or $G_{4}$ & $K=K_{\mathrm{o}}[1-0.9(1-z / h)]$ & $G=G_{\mathrm{o}}[1+9(1-z / h)]$ \\
$K_{5}$ or $G_{5}$ & $K=K_{\mathrm{o}}[1-0.99(1-z / h)]$ & $G=G_{\mathrm{o}}[1+99(1-z / h)]$ \\
\hline
\end{tabular}
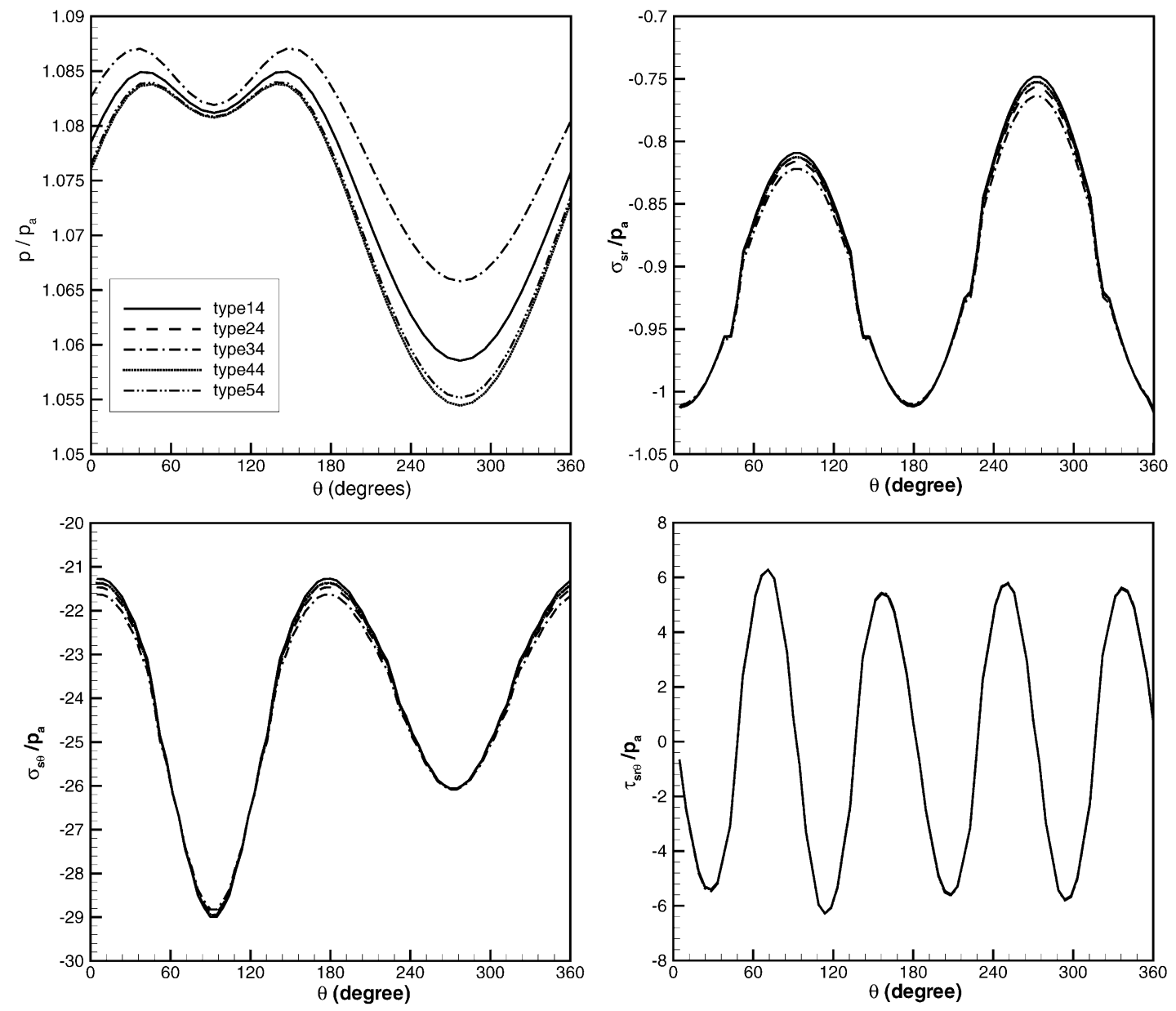

Fig. 5. Distribution of non-linear wave-induced pore pressure, normal stresses and shear stress along pipe surface with variable permeability. 
other parts. But shear stress $\left(\tau_{\mathrm{sr} \theta} / p_{\mathrm{a}}\right)$ is affected very slightly by variable permeability.

It is also observed that the depth functions with same value of $K_{\mathrm{b}} / K_{\mathrm{o}},\left(K_{\mathrm{b}}\right.$ is the permeability of soil near the rigid bottom), such as Types 24 and 44, Types 34 and 54 , significantly affect wave induced pore pressure around the pipe, and that the pore pressure in the seabed with linear reduction of permeability is larger than that with exponential reduction. The wave induced pore pressure in the seabed with variable permeability is higher than that in uniform seabed.

It is noted that the range for pore pressure and internal stresses are different, especially for $\sigma_{\mathrm{sr}} / p_{\mathrm{a}}$ and $\left(\tau_{\mathrm{sr} \theta} / p_{\mathrm{a}}\right)$ (see Fig. 5). The wave-induced internal stresses are much higher than the pore pressure at the surface of seabed.

\subsubsection{Effects of variable shear modulus}

Fig. 6 illustrates the distribution of three-order wave induced pore pressure $\left(p / p_{\mathrm{a}}\right)$, effective normal stress $\left(\sigma_{\mathrm{sr}} / p_{\mathrm{a}}\right.$ and $\left.\sigma_{\mathrm{s} \theta} / p_{\mathrm{a}}\right)$ and shear stress $\left(\tau_{\mathrm{sr} \theta} / p_{\mathrm{a}}\right)$ along pipe surface with variable shear modulus. Unlike the former case with variable permeability, wave induced pore pressure, effective normal stresses and shear stress are
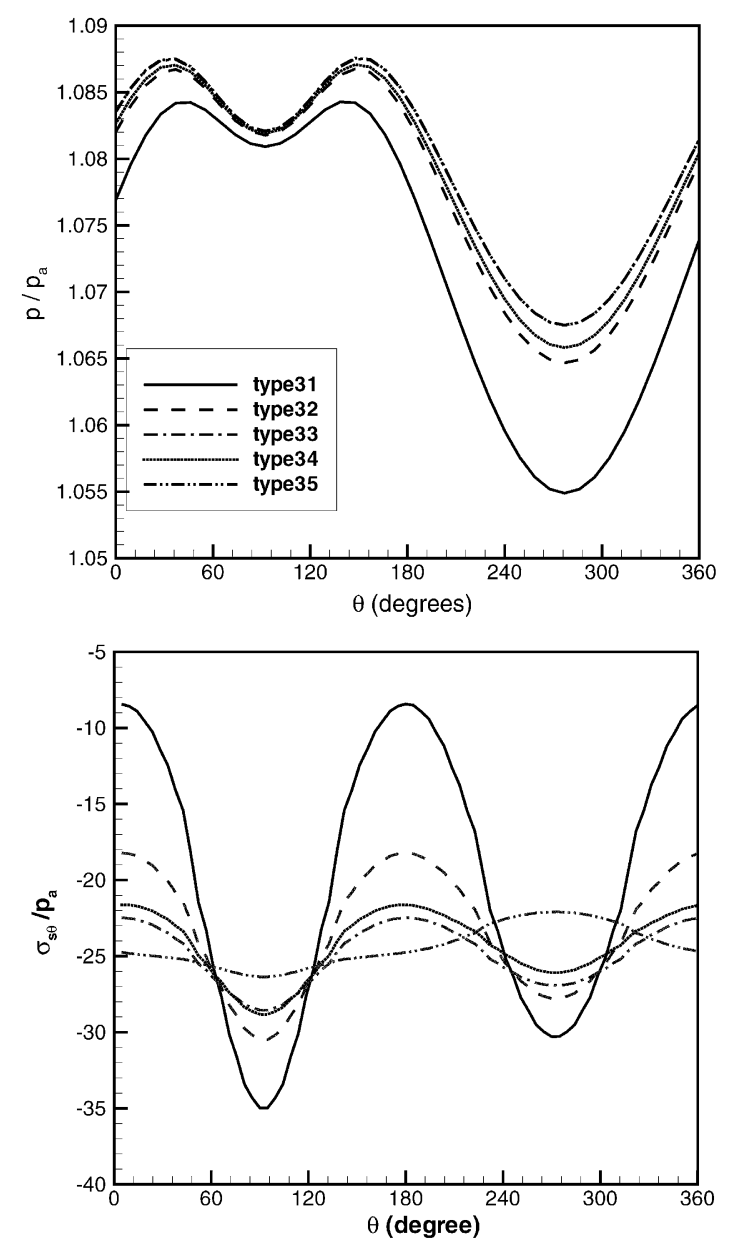

affected significantly by variable shear modulus at any angle $\theta$ around the pipe. The depth function of shear modulus with same value of $G_{\mathrm{b}} / G_{\mathrm{o}}\left(G_{\mathrm{b}}\right.$ is the shear modulus of soil near the rigid bottom), such as Types 32 and 34, Types 33 and 35, significantly affect the internal normal stresses near the surface of pipe, and that the pore pressure in the seabed with exponential increase of shear modulus is larger than that with linear increase. The pore pressure in the seabed with variable shear modulus is higher than that in uniform seabed.

\subsubsection{Internal stresses of buried pipe}

Figs. 3-6 present the soil stresses near the surface of the buried pipe. It is also of interest to examine the distribution of internal stresses within the wall of pipe. Fig. 7 illustrates the distribution of internal stresses $\left(\sigma_{\mathrm{pr}} / p_{\mathrm{a}}, \sigma_{\mathrm{p} \theta} / p_{\mathrm{a}}\right.$ and $\left.\tau_{\mathrm{p} \theta} / p_{\mathrm{a}}\right)$ within the wall. Five different radii $r=0.51894,0.51394,0.50894,0.50394$ and 0.50106 $\mathrm{m}$ are included in the figure. Herein, we used the results for a kind of Gibson Sand (Type 24) and that for a kind of uniform sand (Type 11) as an example. As seen in Fig. 7, the magnitudes of internal normal stress in radial direction $\left(\sigma_{\mathrm{pr}} / p_{\mathrm{a}}\right)$ increase basically as $r$ increases. This implies that approaches zero near the inner surface of
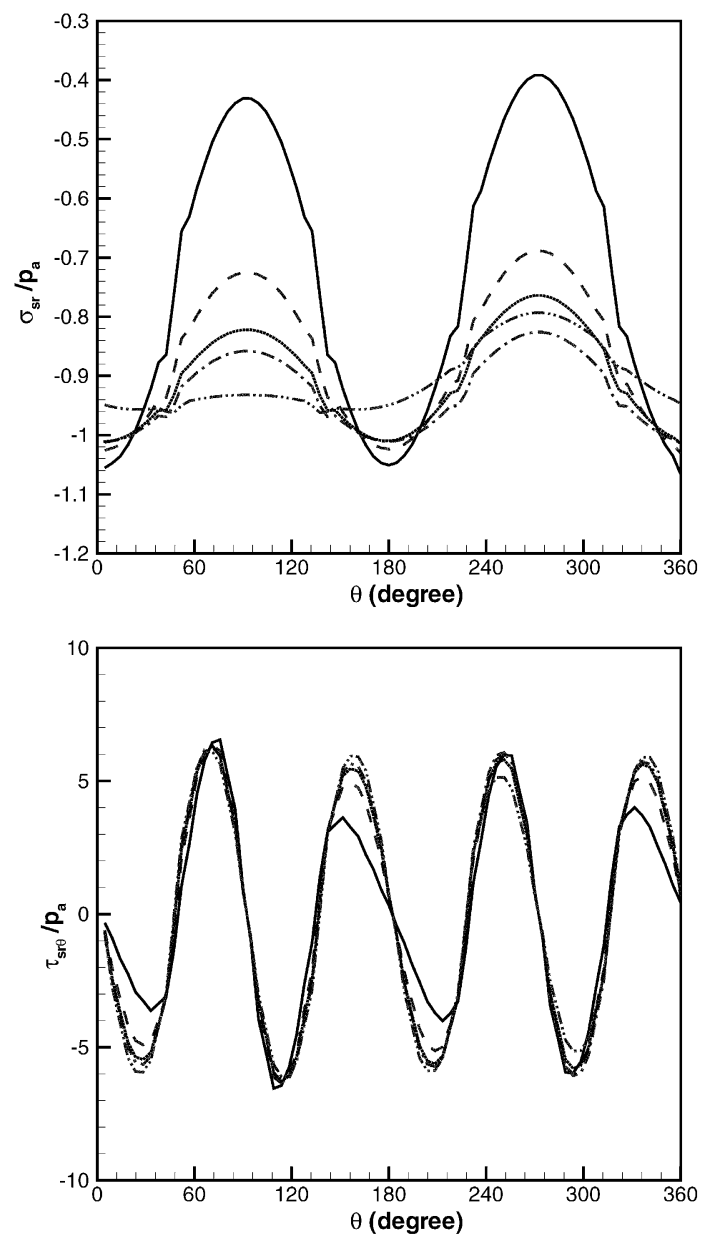

Fig. 6. Distribution of non-linear wave-induced pore pressure, normal stresses and shear stress along pipe surface with variable shear modulus. 

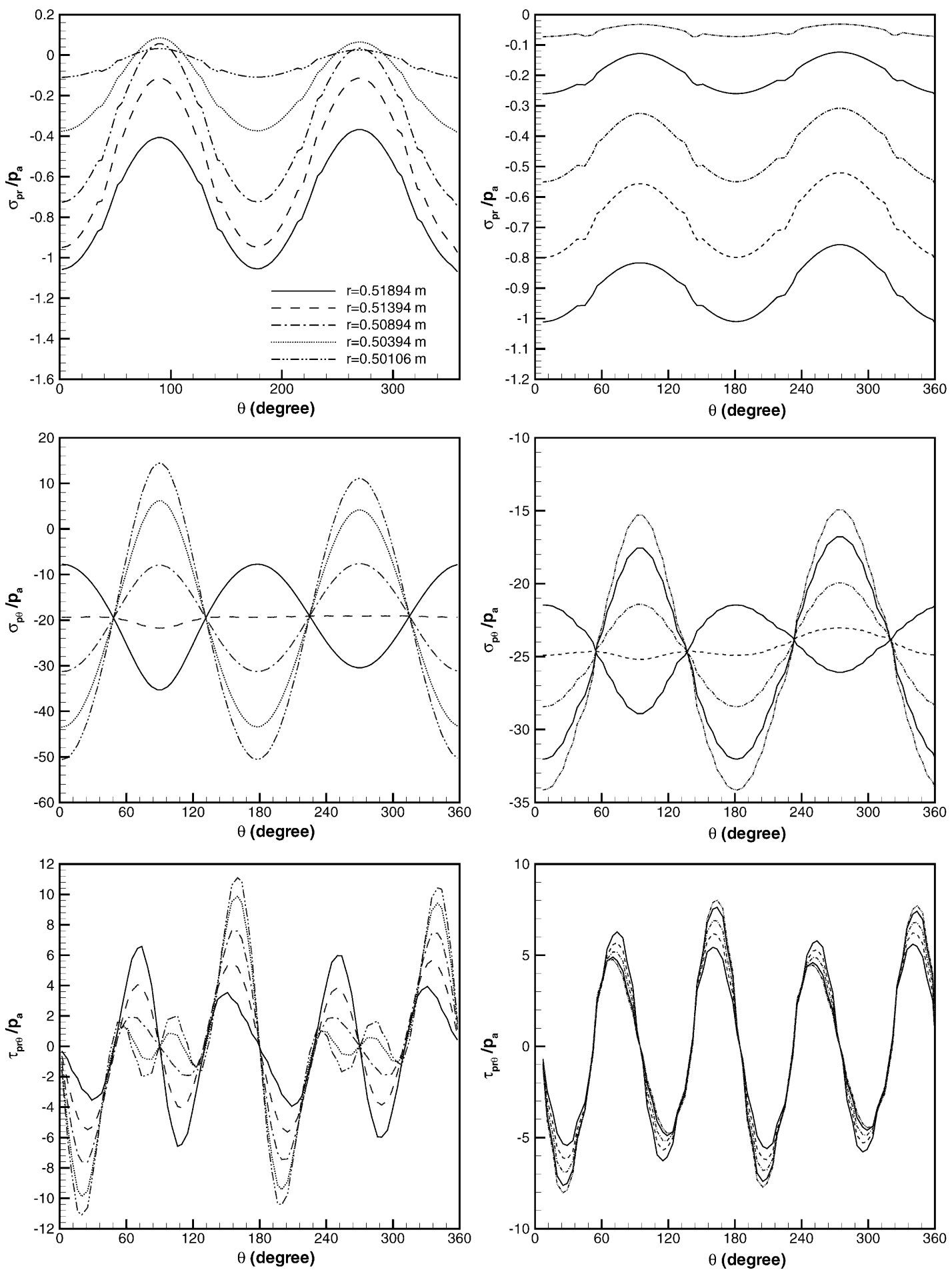

Fig. 7. Distribution of non-linear wave-induced internal normal stresses and shear stress within pipeline (a) Type 11 and (b) Type 24.

the pipelines and increases near the surface of the outer region. The range of the change of for the soil with variable characteristic is much less than that for the uniform soil. The shape of the internal normal stress in angular direction $\left(\sigma_{\mathrm{p} \theta} / p_{\mathrm{a}}\right)$ at separate radius is nearly same for the pipe buried in seabed with variable characteristics (Type 24) as that in a uniform seabed (Type 11). However, the magnitudes of are approximately twice for the soil of Type 24 soil as that for the soil of Type 11 . Therefore, the angular normal stress $\left(\sigma_{\mathrm{p} \theta} / p_{\mathrm{a}}\right)$ is very sensitive to soil characteristics.

\subsubsection{Effects of pipe thickness}

In the aforementioned cases, the pore pressure and internal stresses along the buried pipes with same thickness have been investigated. It is still necessary to 

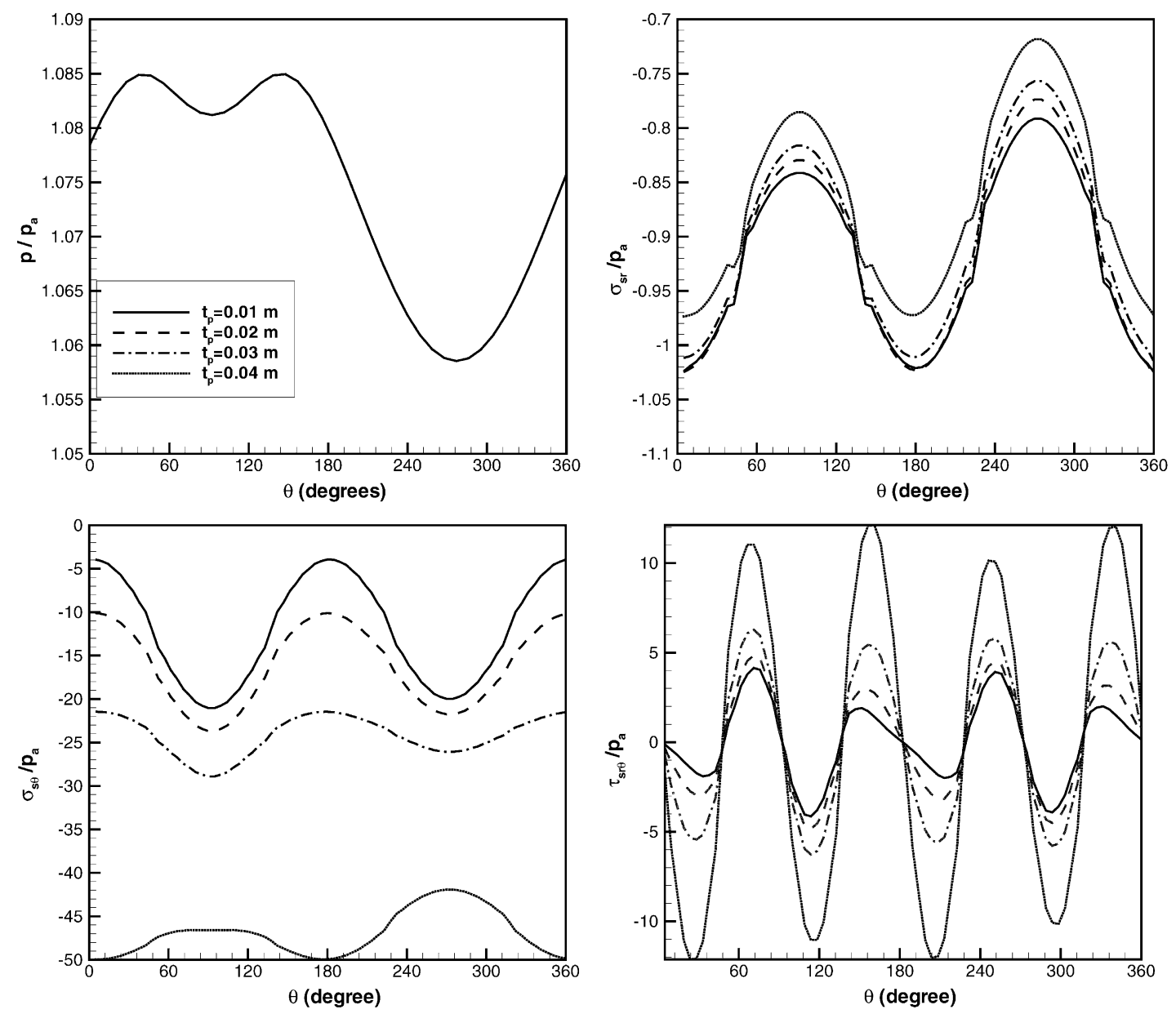

Fig. 8. Distribution of non-linear wave-induced pore pressure, normal stresses and shear stress along the surface of pipeline with various thickness of the pipeline.

study the effects of various pipe thickness on the responses of soil and pipe.

As an example, a kind of Gibson Sand (Type 24) is considered to investigate the pore pressure $\left(p / p_{\mathrm{a}}\right)$ and internal stresses $\left(\sigma_{\mathrm{sr}} / p_{\mathrm{a}}, \sigma_{\mathrm{s} \theta} / p_{\mathrm{a}}\right.$ and $\left.\tau_{\mathrm{sr} \theta} / p_{\mathrm{a}}\right)$ along the pipe with various thickness, as shown in Fig. 8. It can be observed that the variation of pipe thickness $\left(t_{\mathrm{p}}\right)$ from $0.01 \mathrm{~m}$ to $0.04 \mathrm{~m}$ does not affect the pore pressure distribution around pipe. However, $\sigma_{\mathrm{s} \theta} / p_{\mathrm{a}}$ and $\tau_{\mathrm{sr} \theta} / p_{\mathrm{a}}$ are influenced greatly by the various thickness of pipe, and $\sigma_{\mathrm{sr}} / p_{\mathrm{a}}$ is also affected, but much less. Therefore, the slight decrease of thickness may induce a dramatic increase of internal normal stress in angular direction and shear stress within pipeline.

\section{Concluding remarks}

In this paper, the finite element model proposed by the first author [11] is adopted to investigate the nonlinear wave induced soil and pipe response in a porous seabed with variable permeability and shear modulus. Based on the numerical results presented above, the following conclusions can be drawn:

1. The numerical results indicate that the effects of non-linear components on pore pressure and stresses along buried pipeline can not be always ignored without substantial errors, especially for high wave steepness in shallow water.

2. Variable permeability affects wave induced radial normal stress $\left(\sigma_{\mathrm{sr}} / p_{\mathrm{a}}\right)$ much more at the upper and bottom of the pipe than at other parts, but affects angular normal stress $\left(\sigma_{\mathrm{s} \theta} / p_{\mathrm{a}}\right)$ much more at the horizontal sides the pipe than at other parts. However shear stress $\left(\tau_{\mathrm{sr} \theta} / p_{\mathrm{a}}\right)$ is affected very slightly by variable permeability. The depth functions with same value of $K_{\mathrm{b}} / K_{\mathrm{o}}$, significantly affect wave induced pore pressure around the pipe,

3. Variable shear modulus also affects wave induced internal tresses. But, unlike the case with variable 
permeability, wave induced pore pressure, internal normal stresses and shear stress are affected significantly by variable shear modulus at any angle around the pipe. The depth function of shear modulus with same value of $G_{\mathrm{b}} / G_{\mathrm{o}}$ significantly affects the internal normal stresses near the surface of pipe.

4. As shown in Figs. 6 and 7, the wave induced pore pressure in the seabed with variable soil permeability or shear modulus is higher than that in uniform seabed. The non-linear effects are obvious at the upper side of pipeline.

5. The distribution of stresses within pipeline, especially the angular normal stress $\left(\sigma_{\mathrm{s} \theta} / p_{\mathrm{a}}\right)$, is affected by soil characteristics, as shown in Fig. 7.

6. Various thickness of pipe wall influences waveinduced internal stresses too. It is observed that even a slight decrease of thickness may dramatically induce an increase of internal normal stress in angular direction and shear stress within pipeline.

\section{Acknowledgements}

This study was supported by ARC Small Grant (2001) at Griffith University, ARC IREX Award (2001), Australian Academy of Sciences (AAS) and Japanese Society for Promotion of Science (JSPS) Exchanged Program (2001-2002), and Australian Academy of Sciences (AAS) and National Science Council (NSC) Exchanged Program (2002-2003).

\section{Appendix. List of coefficient matrices}

The coefficients $B_{i}(i=1-5)$ in (18), (19) and (22) are listed as follows:

$$
\begin{aligned}
& B_{1}=\left[\begin{array}{llll}
b_{i 1} & b_{i 2} & \cdots & b_{i n_{e}}
\end{array}\right] \text {, } \\
& b_{1 i}=\left[\begin{array}{ll}
\frac{\partial N_{i}}{\partial x} & 0 \\
0 & \frac{\partial N_{i}}{\partial x} \\
\frac{\partial N_{i}}{\partial z} & 0 \\
0 & \frac{\partial N_{i}}{\partial z}
\end{array}\right]=b_{3 i}^{T}, \\
& b_{2 i}=\left[\begin{array}{ll}
N_{i} & 0 \\
0 & N_{i}
\end{array}\right],
\end{aligned}
$$

$$
b_{4 i}=\left[\begin{array}{llll}
\frac{\partial N_{i}}{\partial x} & 0 & 0 & 0 \\
0 & \frac{\partial N_{i}}{\partial x} & 0 & 0 \\
0 & 0 & \frac{\partial N_{i}}{\partial z} & 0 \\
0 & 0 & 0 & \frac{\partial N_{i}}{\partial z} \\
\frac{\partial N_{i}}{\partial z} & 0 & \frac{\partial N_{i}}{\partial x} & 0 \\
0 & \frac{\partial N_{i}}{\partial z} & 0 & \frac{\partial N_{i}}{\partial x}
\end{array}\right],
$$

$b_{5 i}=N_{i}\left[\begin{array}{cccc}1 & 0 & 0 & 0 \\ 0 & 1 & 0 & 0 \\ 0 & 0 & 1 & 0 \\ 0 & 0 & 0 & 1\end{array}\right]$.

The coefficient matrices $D_{i}$ in (18), (19) and (22) are given as

$D_{1}=\frac{\gamma_{w}}{K}\left[\begin{array}{llll}1 & 0 & 0 & 0 \\ 0 & 1 & 0 & 0 \\ 0 & 0 & 1 & 0 \\ 0 & 0 & 0 & 1\end{array}\right]$.

$$
\begin{aligned}
& D_{2}=\left[\begin{array}{ll}
0 & -n \beta \omega \\
n \beta \omega & 0
\end{array}\right], \\
& D_{3}=\omega\left[\begin{array}{ll}
-1 & 0 \\
0 & 1
\end{array}\right],
\end{aligned}
$$

$$
D_{4}=\frac{2 G}{1-2 \mu}
$$

$$
\times\left[\begin{array}{llllll}
1-\mu & 0 & \mu & 0 & 0 & 0 \\
0 & 1-\mu & 0 & \mu & 0 & 0 \\
0 & 0 & 1-\mu & 0 & 0 & 0 \\
0 & 0 & 0 & 1-\mu & 0 & 0 \\
0 & 0 & 0 & 0 & \frac{1-2 \mu}{2} & 0 \\
0 & 0 & 0 & 0 & 0 & \frac{1-2 \mu}{2}
\end{array}\right],
$$

$$
\begin{aligned}
D_{5}= & \frac{2 G_{\mathrm{p}}}{1-2 \mu} \\
& \times\left[\begin{array}{llllll}
1-v & 0 & v & 0 & 0 & 0 \\
0 & 1-v & 0 & v & 0 & 0 \\
0 & 0 & 1-v & 0 & 0 & 0 \\
0 & 0 & 0 & 1-v & 0 & 0 \\
0 & 0 & 0 & 0 & \frac{1-2 v}{2} & 0 \\
0 & 0 & 0 & 0 & 0 & \frac{1-2 v}{2}
\end{array}\right] .
\end{aligned}
$$




\section{References}

[1] Biot MA. General theory of three-dimensional consolidation. Journal of Applied Physics 1941;12:115-29.

[2] Bryant WR, Hottman W, Lambant P. Permeability of unconsolidated and consolidated marine sediments, Gulf of Mexico. Marine Geotechnology 1974;1:1-15.

[3] Cheng AHD, Liu PLF. Seepage force on a pipeline buried a poroelastic seabed under wave loadings. Applied Ocean Research 1986;8:22-32.

[4] Clukey EC, Vermersch JA, Koch SP, Lamb WC. Natural densification by wave action of sand surrounding a buried offshore pipeline. In: Proceedings of the 21st Annual Offshore Technology Conference, Houston, TX, 1989. p. 291-300.

[5] Gatmiri B. A simplified finite element analysis of wave-induced effective stresses and pore pressures in permeable sea beds. Géotechnique 1990;40:15-30.

[6] Gatmiri B. Response of cross-anisotropic seabed to ocean waves. Journal of Geotechnical Engineering, ASCE 1992;118:1295-314.

[7] Gibson RE. Some results concerning displacements and stresses in a non-homogeneous elastic half-space. Geotechnique 1967;17: $58-67$.

[8] Hsu JRC, Tsuchiya Y, Silvester H. Third-order approximation to short-crested waves. Journal of Fluid Mechanics 1979;90:179-96.

[9] Huang NE, Long SR, Tung CC, Bliven LF. A non-Gaussian statistical model for surface elevation of non-linear random wave fields. Journal of Geophysics Research 1983;88(C12):7597-606.

[10] Jeng DS. Numerical modeling for wave-seabed-pipe interaction in a non-homogeneous porous seabed. Soil Dynamics and Earthquake Engineering 2001;21:699-712.

[11] Jeng DS. A general finite element model for wave-seabed-structure interaction. In: Bull $\mathrm{J}$, editor. Numerical analysis and modelling in geomechanics. London: E\&FN SPON; 2003. p. 59-100 [Chapter 3].

[12] Jeng DS, Lin YS. Finite element modelling for water waves-seabed interaction. Soil Dynamics and Earthquake Engineering 1996;15:283-300.

[13] MacPherson H. Wave forces on pipelines buried in permeable seabed. Journal of Waterway, Port, Coastal and Ocean Division, ASCE 1978;104:407-19.

[14] MacDougal WG, Davidson SH, Monkmeyer PL, Sollitt CK. Wave-induced forces on buried pipelines. Journal of Waterway, Port, Coastal and Ocean Engineering, ASCE 1988;114:220-36.

[15] Madsen OS. Wave-induced pore pressures and effective stresses in a porous bed. Géotechnique 1978;28:377-93.

[16] Magda W. On one-dimensional model of pore pressure generation in a highly saturated sand bed due to cyclic loading acting on a sand surface. I: Theoretical description and numerical approach. Internal Report SFB-205, Kusteningenieurwesen, TP A13. University of Hannover; 1990.

[17] Magda W. Wave-Induced uplift force acting on a submarine buried pipeline: finite element formulation and verification of computations. Computers and Geotechnics 1996;19:47-73.

[18] Magda W. Wave-induced uplift force acting on a submarine buried pipeline in a compressible seabed. Ocean Engineering 1997;24:551-76.

[19] Magda W. Wave-induced cyclic pore pressure perturbation effects in hydrodynamic uplift force acting on submarine pipeline buried in seabed sediments. Coastal Engineering 1999;39:243-72.

[20] Mei CC, Foda MA. Wave-induced stresses around a pipe laid on a poro-elastic sea bed. Géotechnique 1981;31:509-17.

[21] Raman-Nair W, Sabin GCW. Wave-induced failure of poroelastic seabed slope: a boundary element study. Proceedings of Institute of Civil Engineers 1991;2(91):771-94.

[22] Sobey RJ, Goodwin P, Thieke RJ, Westberg RJ. Application of Stokes, Conidal and Fourier wave theories. Journal of Waterway, Port, Coastal and Ocean Engineering, ASCE 1987;113:565-87.

[23] Spierenburg SEJ. Wave-induced pore pressures around submarine pipelines. Coastal Engineering 1986;10:33-48.

[24] Thomas SD. A finite element model for the analysis of wave induced stresses, displacements and pore pressures in an unsaturated seabed. I: Theory. Computers and Geotechnics 1989;8:1-38.

[25] Thomas SD. A finite element model for the analysis of wave induced stresses, displacements and pore pressures in an unsaturated seabed. II: Model verification. Computers and Geotechnics 1995; 15:107-32.

[26] Tsai CP, Lee TL, Hsu JRC. Effects of wave nonlinearity on the standing wave-induced seabed response. International Journal for Numerical and Analytical Methods in Geomechanics 2000;24: 869-92.

[27] Yamamoto T, Koning HL, Sellmeiher H, Hijum EV. On the response of a poro-elastic bed to water waves. Journal of Fluid Mechanics 1987;87:193-206.

[28] Zen K, Yamazaki H. Mechanism of wave-induced liquefaction and densification in seabed. Soil Sand Foundations 1990;30:90104.

[29] Zienkiewicz OC, Scott FC. On the principle of repeatability and its application in analysis of turbine and pump impellers. International Journal for Numerical Methods in Engineering 1972;9: 445-52.

[30] Zienkiewicz OC, Taylor RL. The finite element method. 4th ed. London: McGraw-Hill; 1989. 